\title{
ARTÍCULOS
}

\section{EL PAPEL DE LAS MUJERES EN LA TRANSICIÓN CULTURAL DE CUENTEPEC, MORELOS*}

\author{
THE ROLE OF WOMEN IN THE CULTURAL TRANSITION OF CUENTEPEC, \\ MORELOS
}

\author{
María del Carmen Orihuela Gallardo ${ }^{1}$ \\ Centro Regional de Investigaciones Multidisciplinares. \\ Universidad Nacional Autónoma de México
}

Recibido: 3 de diciembre de 2019; Aprobado: 14 de noviembre de 2020.

Cómo citar este artículo / Citation: Orihuela Gallardo, María del Carmen. 2021. "El papel de las mujeres en la transición cultural de Cuentepec, Morelos". Disparidades. Revista de Antropología 76(2): e022. doi: <https://doi.org/10.3989/dra.2021.022>.

\begin{abstract}
RESUMEN: En este artículo se desarrolla la forma en que los diversos factores socioculturales y económicos, que caracterizan la dinámica cultural de la comunidad nahua de Cuentepec, Morelos, definen el papel de las mujeres. Esta comunidad mantiene una identidad apegada a la tradición cultural de los pueblos indígenas que practican la agricultura; sin embargo, en las últimas décadas su dinámica organizacional ha experimentado cambios que muestran una transición socioeconómica. Esto, a partir de la introducción y adaptación de la población a nuevos conectores con el exterior a la comunidad se generó una fluida interacción con la población mestiza. Por ejemplo, con la intensa migración pendular se incrementó la actividad laboral, la profesionalización escolar y la capacidad económica. Si bien, todo esto ha provocado cambios en la interacción comunitaria de las mujeres, es precisamente su actuar social lo que ha permitido mantener la continuidad cultural y la cohesión social interna. Se trata, entonces, de una investigación realizada con metodología etnográfica, con observación participante e interacción directa con la comunidad. Así como de una revisión documental y etnográfica previa.
\end{abstract}

PALABRAS CLAVE: Identidad; Migración; Tradición; Educación; Comadrazgo; Indio.

ABSTRACT: This article develops the way in which the diverse socio-cultural and economic factors, which characterize the cultural dynamics of the Nahua community of Cuentepec, Morelos, define the role of women. This community maintains an identity attached to the cultural tradition of indigenous peoples who practice agriculture; however, in recent decades its organizational dynamics have undergone changes that show a socio-economic transition. The introduction and adaptation of the population to new connectionsoutside the community generated a fluid interaction with the mestiza population. For example, with the intense pendular migration, there was an increase in labor activity, school professionalization and economic capacity. While all this has led to changes in the community interaction of women, it is precisely their social action that has made it possible to maintain cultural continuity and internal social cohesion. It is a question, then, of research carried out with ethnographic methodology, with participant observation and direct interaction with the community. As well as a previous documentary and ethnographic review.

* UNAM. Programa de Becas Posdoctorales en la UNAM, Becaria del Centro Regional de Investigaciones Multidisciplinarias, asesorada por la doctora Úrsula Oswald Spring.

1 Correo electrónico: carmen.orihuela@gmail.com. ORCID iD: <https://orcid.org/0000-0002-5539-2190>. 
KEYWORDS: Identity; Migration; Tradition; Education; Comadrazgo; Indian.

\section{INTRODUCCIÓN}

En la comunidad indígena de Cuentepec, Morelos, se está viviendo una transformación significativa en la vida económica, social y cultural a través de la migración y preparación escolar y profesional de los jóvenes. Las mujeres se colocan como actores sociales determinantes para que estos cambios se ubiquen en un proceso de transición hacia una realidad social y económicamente estable y no a una frágil o desestructurada. Los cambios, entonces, han sido impulsados y soportados por mismas mujeres, no sólo para modificar su propia vida comunitaria, sino también para transformar la condición de vida de sus hijas e hijos y, en general, de las nuevas generaciones.

El objetivo de este artículo es mostrar el proceso social que ha permitido a las mujeres de un pueblo originario generar cambios en las dinámicas sociales reproducidas a partir de valores tradicionales, muchos de los cuales fueron impuestos por prácticas patriarcales y se han reproducido durante generaciones. La importancia del trabajo radica en identificar el importante papel que tienen las mujeres en la reproducción de su cultura ante la generación de un nuevo orden económico comunitario.

Este objetivo se desarrolla con la hipótesis de que los cambios intra-comunitarios se generan por la influencia recibida de proyectos gubernamentales, principalmente, dirigidos a las mujeres de la comunidad. Además, la movilidad poblacional ha permitido una conexión con la cultura del exterior. Así, la transición social en proceso está siendo rápida y evidente, por lo que surgen las preguntas ¿los rasgos culturales comunitarios se disuelven o, en los casos más severos, se destruyen en este proceso? Alternativamente, se puede preguntar ¿cuál es el papel directo de las mujeres en esta dinámica? La respuesta requiere una investigación que fundamente una profunda reflexión etnográfica en esta microcomunidad étnica.

Se empleó una metodología etnográfica y documental. Fue utilizado como fuentes secundarias: por un lado, tesis de licenciatura y posgrados, donde se presenta un trabajo etnográfico en Cuentepec desde 1989 hasta años recientes que desarrollan una descripción de la organización socio-cultural; y por otro, estudios especializados en la cultura y medio ambiente. Asimismo, la investigación fue sustentada con trabajo y metodología etnográfica. El registro de la narrativa local fue el eje que permitió esbozar el papel de las mujeres en el proceso histórico que ha dado dinamismo en la identidad colectiva. Asimismo, se estudió la demografía local, las migraciones, la educación y otras influencias externas en Cuentepec.

\section{CUENTEPEC Y LA VIDA TRADICIONAL DE LAS MUJERES}

Cuentepec es una comunidad nahua localizada en el municipio de Temixco, Morelos en México (Mapa 1$)^{2}$. El nombre de Cuentepec significa "el surco del cerro", nombre que describe el territorio de montañas y tierra surcada para el cultivo. Se ubica en la parte noroeste del Estado, limita con los municipios de Cuernavaca, Miacatlán, Xochitepec, Emiliano Zapata y con el Estado de México. Cuentepec, tiene una superficie de 87.869 km. El Consejo Nacional de Evaluación de la Política de Desarrollo Social (CONEVAL 2012: 33) ubicó la comunidad en un nivel medio de pobreza.

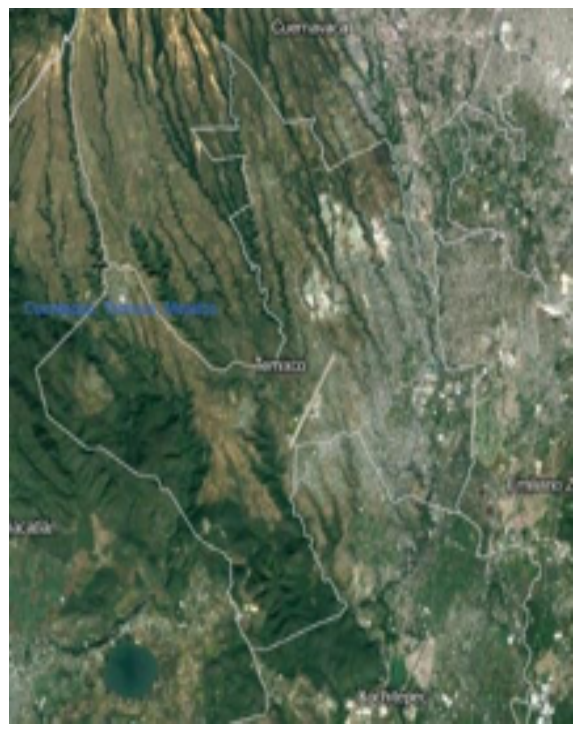

MAPA 1. - Ubicación de Cuentepec, Temixco (INEGI).

2 Sus coordenadas son Latitud $18^{\circ} 51^{\prime} 38.631^{\prime \prime} \mathrm{N}$, Longitud oeste $-99^{\circ} 19^{\prime} 32.663^{\prime \prime}$ W, Altitud 1480 (INEGI). A finales del siglo XX y principios del XXI se estableció que Cuentepec tenía 4,648 hectáreas de superficie ejidal y 2,390 de hectáreas comunales (Paz 2009: 37). 
La población de Cuentepec mantiene una organización social y comunitaria muy apegada a formas culturales definidas como tradicionales. Principalmente, porque la mayoría de sus pobladores se comunican a partir del idioma náhuatl, y las mujeres mantienen el uso de la vestimenta tradicional, que es un vestido satinado con falda plisados al igual que un mandil. En la actualidad únicamente las mujeres adultas la portan, las mujeres jóvenes y niñas, lo tienen en desuso. Portan otro tipo de vestimenta, que generalmente son jeans. El uso del reboso y llevar el cabello largo es lo que permanece. La vestimenta es un indicador del cambio social en las comunidades indígenas (Argón y González 2011: 289).

Hasta hace una década en Cuentepec se mantuvo la práctica endogámica. La población refiere que hombres y mujeres se casaban con personas de la misma comunidad. Incluso, se decía la frase «las mujeres de Cuentepec son para hombres de Cuentepec» (Cruz 2010: 67). Al mantener esta práctica patriarcal, la población conservó su forma tradicional de vida. La frase citada se refuerza con la de "Cuentepec es para los cuentepequenses». El sentido de territorialidad se reafirmaba con la idea de que «si dejamos que vengan otros de fuera, se van a sentir dueños» (Paz 2009: 50). En la actualidad no se acepta que otras personas compren terrenos en Cuentepec. Se piensa que podrían no compartir los principios éticos sobre su territorio, como venderlos a las empresas mineras.

En 1997 se reportó que el total de la población hablaba náhuatl y el $77 \%$ era bilingüe (Manrique 1997: 40). El censo del INEGI muestra que la

\begin{tabular}{|c|c|c|c|}
\hline Año & Mujeres & Hombres & Total \\
\hline 1940 & 461 & 433 & 894 \\
\hline 1950 & 474 & 490 & 964 \\
\hline 1960 & 455 & 532 & 987 \\
\hline 1970 & - & - & 1308 \\
\hline 1980 & 875 & 885 & 1760 \\
\hline 1990 & 910 & 906 & 1816 \\
\hline 1995 & 1314 & 1291 & 2605 \\
\hline 2000 & 1562 & 1543 & 3105 \\
\hline $\mathbf{2 0 0 5}$ & 1797 & 1752 & 3549 \\
\hline $\mathbf{2 0 1 0}$ & 1720 & 1651 & 3371 \\
\hline
\end{tabular}

TABLA 1. - Población entre 1940 y 2010 (INEGI). comunidad había tenido un ritmo de crecimiento pequeño hasta 1970, que comenzó a crecer significativamente. En relación a dicho año, en 2005 la población se había duplicado. En el censo de 2010 se observa que el número de habitantes había disminuido alrededor del $6 \%$ en relación con el periodo anterior (Tabla 1). Esto fue resultado de la migración temporal y definitiva tanto a ciudades cercanas como a Estados Unidos.

Las investigaciones realizadas en la comunidad de Cuentepec identificaron que mujeres y hombres realizaban actividades designadas por el género (Figueroa y Cortes 1989; Manrique 1997; Landázuri 2001; Maestro 2011). En 1997, se registró que las mujeres eran educadas para continuar con los roles establecidos culturalmente en el hogar y el cuidado (Manrique 1997: 40). Las decisiones comunitarias eran tomadas exclusivamente por hombres jóvenes (Figueroa y Cortes 1989: 43).

Cecilia Figueroa y Rocío Cortes (1989: 43) reportaron que a los niños se les permitía jugar por las tardes, lo que no sucedía con las niñas a las que desde los 7 u 8 años se les asignaban tareas como ir a moler el nixtamal. Asimismo, se decía que las mujeres recibían enseñanza y se les dictaban normas para realizar adecuadamente su labor en la vida de pareja. Las autoras mencionaron que «la mujer es siempre tradicional, sigue la escuela de la madre y se forma constantemente por ella» (Ibid: 87). Las actividades productivas de la mujer se centraban en el hogar; su responsabilidad era, y sigue siendo, transmitir valores, costumbres y tradiciones a sus hijos. Ella, la mujer, «entablaba un contacto más estrecho con las niñas, a las cuales inicia y prepara en las labores propias de su sexo que le servirán durante su matrimonio» (Ibid). También se observó que las madres transmitían a sus hijas conocimientos sobre plantas medicinales, elaboración de platillos, crianza y cuidado de los animales de traspatio donde realizan labores de orden económico desde el hogar (Paz 2009: 40).

Todo esto «preparaba a la mujer para pasar directamente a la condición de esposa entre los 12 y 15 años de edad» (Figueroa y Cortes 1989: 86). Alrededor de los 12 años se encaminaba a la mujer a salir para ser cortejadas por los varones, pues se les inculcaba la importancia de casarse jovencitas. Así, en la etapa adulta y una vez casadas, las mujeres, continuaban con las actividades asignadas socialmente de acuerdo con su género. A partir del 
4 de junio de 2019, las leyes nacionales prohíben el matrimonio adolescente (Senado de la Republica 2019). No obstante, los usos y costumbres mantienen la unión de la pareja joven ${ }^{3}$.

Debido a la dificultad para obtener dinero para la manutención de la familia, los hombres se ausentaban todo el día y entregaban el dinero obtenido a sus esposas. Esta dinámica tuvo una relación directa con las dinámicas de violencia a que eran sometidas las mujeres en Cuentepec. Según los testimonios "los hombres traían el dinero" y las mujeres tenían que limitarse a organizarlo bien. Si no lo hacían, les pegaban. De acuerdo con otro testimonio, antes se le pegaba a la mujer en la mayoría de las casas «porque decían que no usaban bien el dinero y ya borrachos comenzaban a pelear». Se dice "ahora ya no es así porque ella también trabaja». Al tener ingresos, producto del trabajo de la mujer fuera de la casa, se comenzaron a modificar las relaciones comunitarias. Los hombres dejaron el machismo y permitieron que las mujeres aportaran recursos a la casa, pues «ellas son muy buenas administradoras y lo invertían en las casas». Además, organizaban los recursos económicos distribuyéndolos en los gastos familiares ${ }^{4}$.

Landázuli (2001: 176) hace ver que las mujeres tenían mucho mayor protagonismo económico, pues si la pareja se separaba, «era la mujer que se quedaba a cargo de los hijos y su manutención, ya que el hombre no aporta ningún apoyo económico». El cambio en la capacidad de las mujeres para obtener recursos monetarios no fue fácil. Se relata que cuando comenzó a haber transporte hacía Cuernavaca ninguna mujer salía a trabajar. «Había mucho machismo. Al principio, si acaso se veía una mujer entre todos los hombres que salían, pero era una mujer viuda o dejada. Porque los hombres no les daban permiso». Continúa el testimonio, «ahora, como las demás mujeres vieron que esa mujer se sabía administrar y se veía su progreso, ellas comenzaron a salir también» (Ibid).

Únicamente los hombres tenían control de la tierra comunal y la decisión sobre ésta. Las actividades en la comunidad se realizan de acuerdo con el ciclo

3 Registro en campo del 16 de octubre de 2019.

4 Eudocio Bello. Ayudantía Municipal de Cuentepec, Mor. Octubre de 2019 agrícola que va desde los primeros días de mayo, para la preparación del terreno, hasta los primeros días de diciembre, cuando se cosecha el maíz. En los tiempos de secas el territorio de Cuentepec cambia drásticamente, deja de ser verde para convertirse en un lugar muy seco (Alavés 2010). Debido a esto, las actividades están asociadas con el momento del ciclo agrícola; las actividades asignadas tanto a hombres como mujeres están asociadas a dicha dinámica. Ellas trabajan en las labores comunitarias para apoyar la actividad familiar, que en la mayoría de las veces son las actividades relacionadas con agricultura. Durante el período de cultivo las mujeres están en casa, donde elaboran los alimentos que el hombre llevará a su jornada en la milpa. En ocasiones ellas les llevan el almuerzo. Dentro de la dinámica milpera, ellas tienen otra tarea: "los hombres van a trabajar la milpa o trabajan en "la obra" o albañilería, pero son las mujeres las que terminan el trabajo para consumo o la venta $»^{5}$. Como sucede en la actualidad, los hombres, para adquirir recursos monetarios, suelen dejar a las mujeres la limpieza, organización y preparación de los productos de la milpa para la venta. Por ejemplo, al frijol, que le quitan la vaina; y la hoja de la mazorca, la organizan en montones. Lo mismo sucede con los productos elaborados como el barro, la cestería, las plantas medicinales y las escobas. Ambos buscan los materiales en el monte, pero son ellas quienes los venden. Con excepción del barro para la alfarería, pues tradicionalmente su recolección es realizada únicamente por mujeres. Ellas venden su producto en Jojutla, Puente de Ixtla, Miacatlán o Cuernavaca, municipios aledaños (Ibid).

Una vez recuperada la producción de los diferentes elementos de la milpa, $70 \%$ de la producción es vendida en Xoxocotla, Temixco, Alpuyeca y Cuernavaca y otras poblaciones del Estado de Morelos; $30 \%$ se destina al consumo familiar (Figueroa y Cortes 1989: 45). Las personas tienen registrado en la memoria que, desde mediados del siglo $X X$, iban a vender sus productos a dichas ciudades trasladándolos en caballo. Don Emiliano recuerda que su abuela de 92 años, para vender el maíz, caminaba con su caballo hasta Cuernavaca o Temixco, entonces considerados pueblos ${ }^{6}$.

5 Eudocio Bello. Ayudantía Municipal de Cuentepec, Mor. Septiembre de 2019.

6 Emiliano Domingo, Cuentepec, Mor. Septiembre de 2019. 
Nubia Maestro (2011: 80) identificó que una de las actividades que mayor impacto en la vida comunitaria, por su requerimiento de energía y tiempo, era lavar la ropa. Don Emiliano, por su parte, relata que se ocupaba alrededor de una hora bajar al río, pero si se quería ir por agua limpia se tenía que llegar al ojo de agua que estaba a hora y media del pueblo. De forma que entre ir a lavar la ropa y ayudar en las labores domésticas, la madre impedía a la niña a asistir a la escuela. Maestro (Ibid) registró este testimonio.

«Yo quería seguir estudiando y ya no me dejaron pues... porque tenía muchos hermanitos, también tenía que hacer quehacer, tenía que ir a lavar al río y no me dejaron pues, fui a la escuela un año... después mi mamá ya no me dejó, si me iba a la escuela ya no me daba para lo que me iba a comprar, me dijo: si quieres ir a la escuela vete pero no te voy a dar para que comas, le dije: no importa yo me voy, y me iba... hacía mi tarea a escondidas, porque mi mamá no me dejaba, se iba con su hermana y me ponía a hacer mi tarea y cuidadito no estuvieran hechas las cosas de la casa... no más aguanté un año porque después me iba a trabajar».

Muchas mujeres adultas coinciden con este testimonio, además agregan que lloraban para poder ir a la escuela, pero no se les permitía porque «no había dinero».

\section{LA IDENTIDAD DE LA COLECTIVIDAD}

Usualmente, en el ámbito antropológico e histórico, se ha interpretado que las sociedades indígenas han logrado preservar sus formas de vida tradicionales gracias a que han generado una cultura de resistencia que ha adoptado una forma de lucha "subterránea o cotidiana» hasta "la resistencia frontal» (Smeke 2000: 92). Sin embargo, en esta interpretación tan difundida de lo tradicional como estático, la población de Cuentepec muestra que su cultura es dinámica y cambiante. En las últimas dos décadas se han presentado procesos económicos, sociales y culturales que han acelerado procesos de cambio.

Gilberto Giménez señala que en la transición de los grupos tradicionales hacia sociedades modernas se producen cambios culturales e identitarios hacia formas individualistas, encuentra un «debilitamiento de las solidaridades sociales» (2010: 14). Ante esto es posible plantearnos la cuestión de si la identidad de los grupos tradicionales, que se configura como colectiva, está en transición hacía una configuración social basada en identidades individuales.

Dice Giménez que «la cultura es la fuente de la identidad "; la cultura es el elemento elemental que marca la distinción entre grupos (Ibid). Por tanto, la manera para hacer el análisis de la colectividad en transición es preciso partir de una cuidadosa observación de las características de la cultura de la comunidad o colectividad (en este caso de Cuentepec). Para esto se podría partir de las categorías que marcarían la identidad de pueblos originarios; de lo que menciona Giménez, la «identidad es definida por otros, en particular por aquellos que se arrogan el poder de otorgar reconocimientos «legítimos» desde una posición dominante» (Ibid). Esta población, Cuentepec, se ha definido a partir de cómo la ven los otros en una «identidad de espejo». Afirma Giménez «este proceso no es estático, sino cambiante» (Ibid). Giménez enlista cinco componentes de la identidad cultural: 1) atributos caracterológicos, 2) estilo de vida relacionado con hábitos de consumo, 3) red personal de relaciones íntimas, 4) el conjunto de objetos entrañables, y 5) por la biografía personal. El autor señala que estos puntos pueden ser relacionados con la identidad individual o colectiva (Giménez 2010: 6-7). Este punto es fundamental, puesto que se aborda la identidad colectiva. Sin embargo, es necesario reconocer que dentro de ese grupo las formas de identidad están fortalecidas por el actuar individual que se dimensiona a formas colectivas de identidad.

Giménez (2010: 7) ofrece algunos de los principios que permiten dimensionar la identidad colectiva. Al citar a Scilla (1983), el autor menciona que esta identidad tiene "la capacidad de diferenciarse de su entorno, de definir sus propios límites, de situarse en el interior de un campo y mantener en el tiempo el sentido de tal diferencia y delimitación». Asimismo, Alberto Melucci, a quien cita Giménez (Ibid), menciona que se debe considerar la identidad colectiva no como una cosa, sino como un sistema de relaciones y representaciones. También, las identidades colectivas pueden pensarse en tres términos: «una definición común y compartida de las orientaciones de la acción del grupo en cuestión»; 
vivir la definición compartida como «modelo cultural», en la cual se incorporan un conjunto de artefactos, prácticas que pueden ser rituales; una estabilidad en la "autodefinición identitaria» (Ibid). Acompañada de un reconocimiento social, las identidades colectivas obligatoriamente deben referirse a "su dimensión relacional» (Ibid). Es decir, se plantea su identidad a partir del reconocimiento de los que no se es. Se encuentra afinidad con los semejantes y se separa de los distintos.

Desde una perspectiva histórica se observa que los grupos étnicos pueden modificar rasgos fundamentales de su cultura, como por ejemplo su religión o su lenguaje, y continuar identificándose como los mismos. De forma que, apunta el autor, «la conservación de las fronteras entre los grupos étnicos no depende de la permanencia rígida de las culturas» (Giménez 2010: 9). Cada pueblo es distinto uno del otro, aunque tengan elementos comunes como la religión. Por ejemplo, dice Miguel Bartolomé (1997: 126) que la identidad debe abordarse dentro de un proceso histórico. Esto lo reafirma Giménez (2010: 10) con las siguientes palabras «las culturas están cambiando continuamente por innovación, por extraversión, por transferencia de significados, por fabricación de autenticidad o por "modernización", pero esto no significa automáticamente que sus portadores cambien de identidad».

La identidad se crea a partir de una posición relacional ante otros grupos. De acuerdo con esto, explica Giménez (Ibid), la «identidad campesina», por ejemplo, será congruente con una posición subalterna que está cargada de valores sociales, por los que se guiara a partir de principios como: «no creerse más de lo que uno es». Dice el autor que las diferencias en las posiciones, que vimos "fundan la identidad", se presentan de dos formas: la objetiva, que es independiente a lo que se piense de ésta; y la subjetiva, que se presenta bajo las "representaciones» que "los agentes se forjan de las mismas».

En las sociedades modernas con formas culturales diversas, dice Giménez (2010: 11), se caracterizan por la "pluralización de las formas de vida», lo cual refiere a una nueva circunstancia social.

«Tal pluralización no podría menos que acarrear consecuencias para la configuración de las identidades sociales. Por ejemplo, cuando el individuo se confronta desde la primera infancia con "mundos" de significados y definiciones de la realidad no sólo diferentes, sino también contradictorios, la subjetividad ya no se dispone de una base coherente y unitaria de donde arraigarse, y en consecuencia la identidad individual ya no se percibe como dato o destino, sino como una opción y una construcción del sujeto».

La identidad «no es más que la representación que tienen los agentes (individuos o grupos) de su posición (distinta) en el espacio social, y de su relación con otros agentes (individuos o grupos) que ocupan posiciones diferenciadas en el mismo espacio» (Giménez 2010: 12). En la relación con otros grupos se busca comprender de qué forma las fronteras de la identidad aceptan nuevos elementos, conservan así sus propios rasgos identitarios. Estas posturas permiten tener más elementos analíticos para comprender la transición a otras formas culturales que repercutirán en la identidad étnica.

\section{SOBRE LAS CATEGORÍAS DE INDIO, INDÍGENA Y PUEBLOS ORIGINARIOS}

Federico Navarrete (2008: 9) refiere que los españoles, a su llegada a América, agruparon la diversidad de los habitantes de las nuevas tierras como indios, se desconocieron las características de cada grupo social. Asimismo, el autor menciona que "la palabra "indígena" deriva de "indio", y con el tiempo ha pasado a significar persona originaria de un lugar. Los indígenas mexicanos son pues, los pobladores originarios de México». A partir de esta última acepción surge la categoría de pueblos originarios, que trae consigo un replanteamiento sobre el trato a los «indígenas actuales» se destaca el origen sobre el proceso histórico que los colocó en una subordinación después de la conquista española.

Reyna Hernández (2013: 94) dice que el concepto de pueblos originarios refiere a los descendientes de aquel pasado anterior a la conquista, pero con una lucha por los derechos de la población cuyo rasgo distintivo en el presente está en su origen, es decir, mantienen la noción de «un pasado y un presente mesoamericano».

En el México independiente se comienza reconocer que las diferencias entre pueblos corresponden a «circunstancias históricas y sociales». Actualmente, la 
pertenencia a un grupo social «indígena» se establece en criterios como la residencia, la participación en comunidad, el lenguaje, la vestimenta y propia autodefinición (Navarrete 2010: 89 y 90).

Federico Navarrete (2010: 172) ha documentado que desde la colonia se creó el concepto de indio para imponer el dominio español, que homogeneizaba a toda la población como inferior. Asimismo, la categoría de mestizos fue adquiriendo diferentes matices durante varios momentos de la historia, pues durante el México independiente se establecía para crear una nueva categoría que definía a la población que dejaba de ser indígena y que tampoco era española (Navarrete 2004: 7). El mestizo se categoriza como el mexicano que ha dejado lo indígena y que mantiene un proceso dinámico e histórico hacia la occidentalización (Ibid: 68). Hasta épocas muy recientes se ha planteado que es artificioso e incluso agresivo contra su propia persona y comunidad, la identificación como indio o indígena.

Navarrete (2010: 172) identifica que en la historia de México es determinante la presencia de las sociedades indígenas para fundamentar y mantener relaciones de dominio que se establecieron en la construcción de un orden político y económico jerarquizado. El agrupamiento de todos los pueblos indios en un concepto de igualdad entre ellos, sin distinguir sus las conformaciones sociales o lenguas de cada grupo. Con todas esas acciones establecieron su dominio y justificaban con esto sus acciones represoras contra los indios. De forma que se crearon múltiples conceptualizaciones en la relación de dominio y subordinación que se interiorizaron profundamente en la mentalidad del mexicano para autodefinirse. En esta definición no se considera lo indio por lo que hay un rechazo a ello.

Según propone Bonfil Batalla (1995: 123), el utilizar la categoría de indio o indígena nos remite a un origen histórico que da lugar a una relación diferenciada entre los colonizadores y colonizados, así se genera una relación dicotómica de subordinación. A partir del punto de vista del colonizador se genera un discurso que busca definir al otro a partir de categorías propias del mismo colonizador. De manera que la posición y la definición de la mujer indígena implicaría, en primer lugar, la carga valorativa e identitaria que la palabra indígena ha conllevado históricamente; en segundo lugar, la carga identitaria y valorativa que la palabra mujer ha conllevado históricamente.
Navarrete (2010: 98) explica la configuración de los conflictos étnicos de la siguiente manera:

"Las realidades étnicas y las realidades nacionales se construyen a través de un diálogo se construyen a partir de un diálogo complejo y dinámico entre la tradición y las realidades actuales, entre las construcciones narrativas del pasado y las expectativas de acción a futuro, y por lo tanto están firmemente ancladas en el tiempo y a la vez se modifican para responder a los cambios y las nuevas realidades».

Según este proceso histórico, lo indígena se vio como la otra parte de la cultura nacional que debía ser asimilada o desaparecida por su atraso cultural, social y económico. Es decir, lo indígena era el par dicotómico del blanqueamiento que exigía el mestizaje, pues expresaba lo peor: pobreza, atraso, ignorancia y fanatismo. Sin embargo, es tiempo de discutir con la visión de los pueblos originarios construida históricamente.

\section{INDIOS, INDÍGENAS Y MACEHUALES: CATEGORÍAS DE IDENTIDAD IMPUESTAS}

Concebir a lo social desde lo multicultural implica el reconocimiento de la existencia de las diferencias culturales (Giménez 2017: 193). Atendiendo a una de las críticas que puntualiza Giménez a ese concepto:

«Justamente por celebrar la diferencia como valor en sí mismo, el multiculturalismo tiende a especializar y congelar las culturas $y$, por lo tanto, las identidades colectivas, asignándoles fronteras claras y a veces rígidas, lo cual contradice la experiencia etnográfica que constatan la fluidez constante de las culturas y la porosidad de sus fronteras» (Ibid: 195).

Dar peso a las fronteras es fundamental en este estudio, pues dentro de las fronteras se encuentran las características de la cultura. Sin embargo, la cualidad de la frontera no rígida es permitir entrar elementos culturales de otros grupos sociales conservándose la identidad propia.

Para aproximarse a este proceso social de transferencia de elementos identitarios el concepto de transculturalidad resulta relevante. Dice Giménez, al retomar a Demorgon (2005), que esta categoría 
de análisis refiere a "lo que transita de una cultura a otra: una modalidad alimentaria o vestimentaria, un rito religioso o una técnica» (Giménez 2017: 204). Transculturación remite, en una forma activa, al proceso de transferencia cultural, «a la acción y el efecto de trasferir rasgos o elementos culturales de una cultura a otra» (Ibid: 205). Lo transcultural, funciona como elemento articulador para mantener unidos a grupos distintos sin violencia (Ibid).

Hemos referido antes que los grupos étnicos continúan en una dinámica de dominación subordinación, donde han conformado su identidad en torno a dichos conceptos. Sin embargo, en el caso de Cuentepec, en el proceso de transculturación surge una necesidad émica, es decir, conocer «la imagen de la cultura dominante que tienen los subordinados", (Giménez 2017: 215). Esto cobra importancia porque en el discurso de Cuentepec, pues existe una imagen del grupo externo a la comunidad. Anteriormente, se llamaba koyome (coyote) a los de fuera, porque ellos "comen carne" o son agresivos y pueden hasta "matar», recuerda a los soldados que llegaban al pueblo durante la Revolución 7 . Hoy en día se les llama "comare o compare», término que refiere a los que no son hablantes de náhuatl (Domingo 2016: 25). Al ser una referencia amable, estas últimas categorías marcan una transición en la forma de pensar a los otros.

La mujer indígena, por definición, es parte inseparable de un colectivo, de una comunidad. Esto se contrapone con la idea de la individualidad como opuesto a la comunidad. A la mujer indígena se le presiona desde la sociedad dominante para abandonar sus costumbres como forma de emancipación. Pero ¿quién debe cambiar? ¿la sociedad hegemónica que no acepta lo diferente - las mujeres indígenas que están profundamente integradas a su vida comunitaria? Intentar comprender el significado de lo que representa ser «mujer indígena» exige una apertura epistemológica que permita una ubicuidad más amplia que los modelos binarios preestablecidos de género, que remiten a la vulnerabilidad y a la agresividad hacía las mujeres.

Hoy en día la población nahua de la comunidad de Cuentepec se nombra con la palabra «indio»

7 Testimonio de un habitante de Cuentepec, junio de 2019. o "macehual»". Ambas categorías responden al proceso histórico que los ubicó socialmente en una posición de subordinación ante el grupo dominante. Porque esta categoría refiere a la forma en que los españoles y después mestizos llamaban a la población étnica, quienes lo asumieron como una forma relacional.

Alfonso Caso (1948) señaló que eran cuatro aspectos que identificaban a las personas como indios: el biológico, el cultural, el lingüístico y el psicológico. Este último es el más sensible pues refleja la importancia de que una persona se sienta parte del grupo social llamado indio o indígena. Esto remite a diferentes circunstancias. Una de las características, acaso definitorias, que asumen las personas de un grupo social, que es denominado como indio, es su sentimiento de pertenencia a una comunidad. Lo que conlleva a la identificación con los valores culturales de ésta, que van desde el uso de la lengua indígena hasta la práctica de múltiples formas tradicionales de la cultura. De manera que la pérdida de las redes sociales con otros miembros del grupo implica un proceso de des-identificación y de cambio de identidad. Esto con las problemáticas y conflictos sociales que conlleva.

En ese mismo sentido, aunque no de la misma forma, una mujer de Cuentepec dice no saber por qué a ellos se les define como indígenas, o indios. Incluso, ellos, en la comunidad no se identifican como nahuas, como se les reconoce entre la población fuera de la comunidad. Con el paso del tiempo, y con la enseñanza en las escuelas, saben que su idioma es llamado náhuatl y que se les identifica como indígena ${ }^{9}$. Sin embargo, los cuentepequenses, después de la imposición de los últimos años, ya lo reconocen así. Esto nos habla de que las mujeres de grupos sociales distintos tienen que entender las categorías impuestas y aceptarlas, sin identificarse con ellas.

Lo mismo con categorías como macehual. Macehual es referido en las fuentes como merecer (Paredes 1579). Miserable /pobre / vasallo (Tezozomoc 1578), acaecer en desdicha (Molina 1571) ${ }^{10}$. Las traducciones de los diccionarios

8 José Luis Torres. Cuentepec, Mor. Mayo de 2019.

9 Maribel Villegas. Habitante de Cuentepec, Mor. Maestra de náhuatl en Cuernavaca. Agosto de 2019.

10 Véase en Gran diccionario Náhuatl. IIB y IIH, UNAM, 2018. <http://www.gdn.unam.mx> 
de náhuatl de los españoles dan cuenta de la forma de entender a la población que trabajaba en las haciendas. Sin embargo, este término continúa siendo un referente identitario para los cuentepequenses, pues dicen «nosotros sabemos que somos macehuales» ${ }^{13}$. Ante esta frase común en Cuentepec, Maribel Villegas explica que se les llamó así porque se les identificó como aptos para realizar trabajos duros. Sin embargo, ella se opone a esa designación al decir que «no sólo somos gente para trabajar, tenemos inteligencia. No somos sólo personas que sólo sirven para trabajar».

Maribel expresa: «[...] así, lo dicen porque ven nuestras manos resecas por el trabajo que hacemos. Se piensa que por las características de nuestras manos sólo estamos para trabajar ${ }^{11}$. En lo dicho por Maribel encontramos que las mujeres generan un discurso para defender su identidad interna $y$, de esta forma, no continuar con lo que se impone desde lo externo. Su discurso, como mujer, muestra el dolor que esto le genera al pensar en su comunidad. Esto porque al asociarlas con las categorías que se derivan de lo indígena, comienza la discriminación a lo que representan conceptualmente para la población mestiza de México.

\section{LA TRANSICIÓN SOCIOECONÓMICA EN CUENTEPEC}

Para planear una transición sociocultural en Cuentepec parto de que se trata de un pueblo originario con una dinámica cotidiana apegada a la tradición agrícola, que se ha incorporado a la economía y estructura socio-política ligada al proyecto de nación. Esta transición no refiere necesariamente a una pérdida o transformación de las formas sociales ligadas a aquella tradición de la que se partió. Más bien, la organización social comienza situarse dentro de un proceso de transición comunitaria, en el que se presentan cambios en la dinámica social cotidiana.

En esta dinámica de transición se observan repercusiones económicas y sociales en las comunidades indígenas que entran en contacto e influencia con un grupo dominante. Benavides (2011: 23) refiere que «la justicia en épocas de transición es vista como una utopía realizable, pues permite a las

11 José Luis Torres. Habitante de Cuentepec, Mor. Mayo de 2019 sociedades movilizarse y actuar, pese a la existencia de los múltiples desafíos que enfrentan los estados frágiles o en situaciones de fragilidad» Las sociedades que entran en contacto se influyen, pero el que más puede modificar su cultura es el grupo más frágil.

En la dinámica generada a partir de los cambios económicos y políticos se generan actividades para cumplir con las demandas emergentes. Según menciona Orjuela (2003: 55), la reestructuración económica cambia las reglas que regulan las relaciones sociales. Según la propuesta del autor, la política y la economía generan un conjunto de reglas que dan forma a la interacción social, pues «proporcionan una estructura a la vida diaria y, por lo tanto, definen y limitan el conjunto de acciones y elecciones de los individuos» (Orjuela 2003: 54). Se observa entre las mujeres de Cuentepec una importante capacidad para transitar entre los cambios y continuidad de las dinámicas identitarias.

\section{LOS CAMBIOS QUE SE PRESENTAN EN CUENTEPEC}

El proceso de transición que vive la comunidad de Cuentepec ha estado marcado por tres momentos: la construcción de la carretera a principios de la década de los noventa; la construcción del bachillerato en la comunidad en 2001; y la constante integración e impartición de diferentes apoyos y proyectos de investigación dirigidos hacia las mujeres (González 2005). Desde de la década de los 90 se observó la constante participación de las mujeres en proyectos del DIF, donde aún se preparan desayunos escolares, y reparto de despensas donadas por el INI, por ejemplo (Figueroa y Cortes 1989: 43).

Cuentepec ha sido receptora de múltiples talleres o programas sociales gubernamentales. Por ejemplo, la Secretaria de Desarrollo Social (SEDESOL) ha promovido talleres de alfarería que es una de las actividades tradicionales. El Ayuntamiento de Temixco ha promovido talleres de costura. La Comisión para el Desarrollo Indígena (CDI), de huerto familiar. La Facultad Agropecuaria de la UAEM, sobre elaboración de lácteos y dulces, entre otros. De todos los talleres impartidos el que tuvo mayor participación y continuidad en la comunidad fue el de alfarería, ya que la motivación para realizarlo y continuar con la actividad era la satisfacción de realizar una actividad de larga tradición vigente en 
el pueblo y que, además, permite a las mujeres permanecer en la comunidad. La alfarería es un trabajo propio de las mujeres, únicamente ellas pueden acudir a las cuevas para extraer la materia prima. Actualmente, son 40 mujeres las que trabajan con el barro, por ello el Instituto Mexicano de los Pueblos Indígenas (INPI), y el Ayuntamiento de Temixco, apoyan a las mujeres con programas para su promoción. Incluso, se ha creado un grupo de mujeres alfareras llamado Mujeres de Barro (Suazokitl).

En los otros talleres, por ejemplo, la elaboración de queso solamente una persona continuó con el trabajo aprendido por representar una ganancia económica (Martínez 2016: 40-41). El no continuar con actividades como el huerto en patio trasero, la elaboración de dulces y quesos tiene que ver que los instructores no regresan para darle continuidad a terminar y reforzar la capacitación y que las mujeres decían no tener herramientas adecuadas para ello (Ibid). El abandono de estos talleres es porque no tiene tampoco sentido tradicional en la población. Las mujeres jóvenes prefieren estudiar una carrera o «trabajar en otra cosa» (Ibid).

La misma población asocia los cambios generados en la comunidad con la apertura de la carretera que generó un importante flujo migratorio a la ciudad de Temixco (40 minutos) y Cuernavaca (dos horas). La «migración péndulo o temporal» ${ }^{12}$ a centros urbanos es una de las principales alternativas económicas de la población (Torres 2018: 8). Se registró que el 80 $\%$ de los hogares tenían al menos una persona que migra por un día o por varios a dichas ciudades (lbid). Muchas mujeres adultas, portan su vestimenta tradicional $y$, con esta, se trasladan a Cuernavaca para vender sus productos en el mercado, como ha sido la costumbre desde décadas anteriores. Entre los productos se encuentran escobas o frijoles frescos o secos, frutos rojos o quelites que crecen en las milpas en temporada de lluvias. También, venden ciruelas o cacahuates. Actualmente se observa un incremento en la migración de las mujeres de entre 17 a 50 años, pues ellas se trasladan principalmente para emplearse en el trabajo doméstico de entrada por salida o de planta en los municipios aledaños.

12 Es definida como la movilidad al lugar de trabajo que implica la salida y regreso el mismo día (UNESCO). <http://vocabularies.unesco.org/browser/thesaurus/ en/page/concept5884?clang=es $>$.
Diariamente salen de la comunidad alrededor de 540 personas en 11 camiones de línea, además en diversos autos particulares que parten llenos entre las 5 y 8 de la mañana (Ibid).

En 2005 se registró que la migración al extranjero apenas comenzaba a realizarse. Principalmente migraban jóvenes varones. En los 10 años anteriores habían migrado 109 individuos, de los que 49 regresaron, 45 se quedaron y 5 viajan cada año (González 2005). Posteriormente, los varones se organizaron para viajar en camiones hacia Estados Unidos, pero en los últimos años ante las dificultades para pasar la frontera se ha suspendido ${ }^{13}$.

La migración estaba y, en la mayoría de los casos, se mantiene organizada de acuerdo con los ciclos y actividades agrícolas. Los hombres casados que cuentan con terreno de cultivo continúan teniendo como actividad principal el trabajo de la milpa. En esta lógica, durante la época de lluvias, salen de las casas para cuidar el cultivo. La agricultura y la diversificación de las actividades secundarias al trabajo de la milpa continúan siendo las más importantes para las mujeres de la comunidad de Cuentepec (Martínez 2010: 11). La elaboración de productos con materiales obtenidos en su entorno natural ha representado históricamente una alternativa económica para las familias. Esta dinámica económica es una de las formas más arraigadas en la vida de la población agricultora. Las mujeres se mantienen en casa o salen de Cuentepec para vender o para trabajar en el servicio doméstico, ya que apoyan a los gastos de la familia con su trabajo durante el periodo de producción agrícola.

Durante el conocido tiempo de "cuaresma», que abarca desde noviembre hasta mayo, muchos hombres, de entre15 a 50 años, salen de la comunidad para realizar trabajos de albañilería. Esto, porque de acuerdo con Manrique (1997: 40) sus principales actividades son la agricultura, ganadería, albañilería. Los hombres jóvenes, pueden apoyar a sus papás en la milpa, pero al no tenerla a su cargo, o estar alejados de las actividades agrícolas, migran a lo largo de todo el año para realizar trabajos como jornaleros o en la construcción en ciudades cercanas como Temixco o Cuernavaca. En ocasiones, se quedan fuera de la comunidad toda la semana y regresan el

13 Eudocio Bello. Encargado de la Ayudantía del Municipio de Cuentepec, Morelos. Octubre de 2019. 


\begin{tabular}{|l|c|c|c|c|c|c|c|}
\hline & \multicolumn{3}{|c|}{ Ingreso } & \multicolumn{3}{c|}{ Egreso } & Población \\
\cline { 2 - 8 } & Mujeres & hombres & Total & Hombres & Mujeres & Total & total de estudiantes \\
\hline $\mathbf{2 0 1 4 - 2 0 1 5}$ & 34 & 35 & 69 & 19 & 25 & 44 & 142 \\
\hline $\mathbf{2 0 1 5 - 2 0 1 6}$ & 31 & 32 & 61 & 14 & 15 & 29 & 126 \\
\hline $\mathbf{2 0 1 6 - 2 0 1 7}$ & 37 & 21 & 58 & 19 & 23 & 42 & 125 \\
\hline $\mathbf{2 0 1 7 - 2 0 1 8}$ & 21 & 25 & 46 & 14 & 20 & 34 & 106 \\
\hline $\mathbf{2 0 1 8 - 2 0 1 9}$ & 17 & 20 & 37 & 25 & 14 & 39 & 94 \\
\hline
\end{tabular}

TABLA 2.- Relación de estudiantes (COBAEM, EMSAD 02, Servicios Escolares).

fin de semana a Cuentepec. También, pueden irse por varios meses, contratados en estados del norte del país.

Con el acceso a mayores recursos monetarios se generaron cambios determinantes en la transición cultural. Tanto que la migración ha sido un factor para el cambio drástico en la fisonomía del pueblo que, por ejemplo, se muestra en la construcción de casas con materiales no perecederos. Esto generó que las casas no estuvieran dispersas en el lomerío de la zona, sino congregadas.

Por la movilidad provocada por la migración, desde el 2010 en Cuentepec se comenzaron a casar con gente de fuera. La comunidad comenzó a abrirse para incorporarse a la vida del exterior moviéndose de la vida intra-comunitaria hermética que históricamente habían tenido. Se ha permitido contraer matrimonio, tanto hombres como mujeres, con gente de fuera. También hay casos en los que las mujeres salen de la comunidad por matrimonio. Como se mencionó, otro de los cambios que ha tenido un impacto trascendental y que dirigen a la población en Cuentepec hacía una transición social lo marca el establecimiento del plantel Bachilleres EMSAD-COBAEM en 2001. En los primeros tres años del bachillerado hubo únicamente mujeres inscritas, los siguientes ciclos ya se habían inscrito hombres, aunque muchos de ellos se daban de baja. Fue en la generación 2014 que los muchachos se inscribieron de forma equilibrada a las mujeres (Tabla 2). Así se muestra a continuación.

La mayor participación de los jóvenes en los diferentes grados de estudio se dio con la implementación de las becas familiares otorgadas por el programa PROSPERA, que fue implementado desde 2014 el "Programa Jóvenes con Oportunidades», como incentivo a la graduación de la educación media superior (Coordinación Nacional de PROSPERA Programa de Inclusión Social 2018). Después, entre 2013 y 2018 se entregó la Beca Salario en Morelos, que otorgaba el pago de la beca directamente al estudiante de bachillerato desvinculándolo de la economía familiar.

Los jóvenes de Cuentepec tienen acceso a un nivel mayor de estudios. De acuerdo con el seguimiento que se hace de los estudiantes, el $15 \%$ de ellos continúan con sus estudios hasta avanzar y concluir una carrera universitaria. Todos los alumnos que hacen examen de admisión consiguen su ingreso a la universidad. Los jóvenes han optado por estudiar diferentes carreras, no sólo docente en educación indígena como antes ocurría ${ }^{14}$. De manera que, en los últimos años, la migración a la ciudad también está dada por jóvenes, mujeres y hombres, que se trasladan principalmente a la universidad del estado (UAEM) o la Universidad Pedagógica Nacional.

\section{LA DINÁMICA DE TRANSICIÓN EN CUENTEPEC}

Ante los cambios en la fisonomía del pueblo, la obtención de servicios el agua potable, la posibilidad de transportarse por la carretera y estudiar en el bachillerato de Cuentepec los habitantes de la comunidad refieren dos posturas, la que asumen hombres y las mujeres de alrededor de setenta años. Don Emiliano de 65 años, comenta que para él era mejor antes, pues todo se tenía sin necesidad de trabajar tanto; no se pagaba la luz, el agua o el teléfono, además de los gastos de las escuelas. Comenta que «había tiempo para descansar. Hoy en día no hay tiempo para el descanso, ni en tiempo de

14 Ing. Noé Rafael Pérez. Director de COBAEM EMSAD 02 de Cuentepec, Morelos. Octubre 2019. 
cuaresma [secas]». Al tener presente que se debe cubrir los gastos familiares, cada vez más altos, se necesita migrar durante todo el año. Por su parte, los sentimientos de la mujer ante los cambios son distintos, doña Reynalda de 64 años, dice que es mejor la vida ahora, antes la producción en venta se llevaba en caballo, mientras que la persona caminaba; ahora se llevan los productos en camión. Además, comenta doña Reynalda, hoy en día el agua potable llega hasta la casa, cuando antes se tenía que bajar al río a lavar. Había que regresar con la ropa lavada además de un poco de agua para la comida ${ }^{15}$. Las mujeres antes no tenían descaso, aunque, tampoco ahora sólo que las condiciones han cambiado.

Aún con el alto grado de identidad nahua en Cuentepec, son las mujeres quienes se adaptan particularmente a los cambios que se generan en la dinámica social. Las modificaciones sociales por influencia del Bachilleres han ido lentamente. Livia González en 2007 realizó una serie de encuestas aplicadas a las primeras generaciones del bachillerado, compuestas por mujeres, en este estudio se encontró una situación relevante en los cambios que comenzaban a encontrarse, menciona que las mujeres eran las que asistían al bachillerato, no por una búsqueda de cambio de vida, sino porque la mayoría de los hombres estaban dedicados a las actividades del campo o a otras actividades económicas. Asimismo, reporta algunos testimonios de las jóvenes estudiantes ante la pregunta "¿qué querían ser en el futuro?» mencionaban que preferían casarse $^{16}$.

Sin embargo, algunas otras mujeres jóvenes optaron por estudiar. A partir de esta situación comenzaron a caer en cuenta que al estudiar podían desarrollarse y mejorar su economía. Las entrevistas y encuestas no reportan algún resultado sobre la aceptación o rechazo de las mujeres en torno a su cultura o sociedad. Esta situación resulta sumamente importante en el proceso de transición de las mujeres, que les posibilitó ganar una independencia económica y tener una dinámica de vida diferente a la que habían sido preparadas a lo largo de su vida, como reproducir las normas sociales en su conducta. Todas

15 Reynalda Domínguez Marquina. Habitante de Cuentepec, Morelos. Septiembre 2019.

16 Manuscrito de Livia González Ángeles. 2007. «Jóvenes mujeres de Cuentepec, Morelos. ¿Aspiraciones y modelos femeninos en transformación?». relacionadas con roles de género (González 2005). Las normas inculcadas por las madres y aprendidas por sus hijas. Hoy en día se registra en la narrativa que las mujeres son la base de la sociedad de Cuentepec.

En la actualidad puede observarse que las jóvenes, entre los 15 y 24 años, ven con naturalidad la vida en unión libre al igual que los adultos. Se quedan con la promesa de hacer la boda religiosa, pero ésta se pospone por falta de dinero. Se observa también que algunas mujeres jóvenes se "van con su novio» al terminar la secundaria. El unirse con la pareja, o «juntarse», es una costumbre muy común. No obstante, esto no impide que las mujeres sigan con sus estudios apoyadas por sus parejas o/y familias. Incluso, se presentan casos en el Bachillerato en que las estudiantes asisten embarazadas y reciben el apoyo de docentes y padres, principalmente de las madres, que se quedan al cuidado de los niños.

En la vida actual de las comunidades indígenas, las mujeres tienen formas diversas de ubicarse en la dinámica cotidiana. En este contexto de cambio cada mujer vive realidades distintas a la que viven las otras, aunque ellas pertenezcan al mismo grupo social, a la misma edad, o a la misma actividad familiar o económica. Asimismo, las mujeres mayores no pueden pensarse a sí mismas y a su entorno territorial en las mismas circunstancias que la situación de las mujeres jóvenes o las niñas. De manera que hablar sobre la mujer indígena no se debería generalizar su historia, su condición y ubicación geográfica, su labor en su colectividad, su ocupación laboral, su condición migratoria, su edad, etcétera. La situación actual de las mujeres puede ser muy diversa. Considero que estas diferencias se acentúan por las circunstancias diferentes que viven las diferentes generaciones y sus condiciones socioeconómicas y educativas.

En Cuentepec se observa una sólida identidad en las relaciones familiares y comunitarias en la forma de asentar las normas sociales. Las familias transmiten a los niños el idioma náhuatl y los valores sociales y éticos de sus antiguas costumbres. Todos estos elementos culturales transmitidos socialmente se presentan continuamente, aunque se hayan incorporado nuevos a la dinámica social actual. Por ejemplo, las mujeres adultas se conducen de acuerdo con las normas sociales tradicionales, las cuales siguen con determinación. A diferencia de ellas, muchas mujeres jóvenes cuentan con mayor grado de estudios, lo que provoca que ellas cuestionen las mismas normas 
sociales. Pocas veces visten la vestimenta tradicional y manejan el español y el náhuatl con soltura. La incorporación cada vez mayor de los jóvenes a la dinámica escolar es uno de los puntos importantes de reflexión: ¿cómo manifiestan los cambios culturales con el aumento de la transculturación?

Giménez (2010: 6) refiere lo fundamental que pueden resultar en los contenidos de los lazos comunitarios en la conformación de la identidad la red personal de relaciones íntimas, que describe así: "cada quien tiende a formar en rededor un círculo reducido de personas entrañables, cada una de las cuales funciona como "alter ego" (otro yo), es decir, una extensión y "doble" de uno mismo, y cuya desaparición (por alejamiento o muerte) se sentiría como una herida, como una mutilación, como una incompletud dolorosa». Esto muestra que las redes comunitarias, como un rasgo de identidad, son muy fuertes, y existen mecanismos para mantenerlas como parte de sus costumbres. Estas redes se conforman sólidamente y van más allá de ofrecer recursos o alimento a los que lo necesitan.

Así, las sociedades indígenas mantienen una dinámica comunitaria a partir de prácticas para consolidar alianzas y relaciones muy sólidas que se construyen continuamente. Estas relaciones llevan a la comunidad a establecer, mantener y crear redes que permiten la cohesión social y la permanencia cultural. Las prácticas colectivas de reciprocidad generan mayor empatía ante las circunstancias del resto. Así, con acciones contantes de intercambio y reciprocidad se fundamenta la cohesión social (Mota y Sandoval 2011). Estás características se reafirman a través del vínculo de comadrazgo ${ }^{17}$ establecido entre las mujeres.

El comadrazgo es una práctica que refirma relaciones complejas. Livia González describió que por este acto se crean y consolidan redes horizontales que fortalecen los vínculos de apoyo y solidaridad entre las mujeres. Así como las relaciones se hacen fuertes entre hombres a través del compadrazgo, entre mujeres también se crean relaciones de amistad con el comadrazgo (González 2016: p. 44). Es tan fuerte esta relación que ellos llaman «nantzin» (madre con marca de honorifico) a las mujeres con

17 La RAE define comadrazgo como «parentesco espiritual que contraían la madre de una criatura y la madrina de esta». que se establece este lazo. Se puede proponer que con compadrazgo y comadrazgo se crea una relación de parentesco no consanguínea.

En este contexto, las palabras de Maribel Villegas: "somos una hermandad ${ }^{18}$ cobran mayor sentido. Las relaciones parentales, consanguíneas o establecidas socialmente, son un lazo que se crea por medio de diversas ceremonias en las que se entrega un don o un regalo. En particular, el establecer redes a través del comadrazgo busca mantener una protección y cuidado hacia los más jóvenes de la comunidad, y redes de apoyo entre los adultos. La pedida y aceptación, que tiene que ver con la formalización del comadrazgo, ofrece jerarquías que se muestran como prestigio social entre el grupo de mujeres. En Cuentepec se observa que las madres, comadres y ahijadas se apoyan de diferentes formas. Por ejemplo, en la participación en las fiestas con regalos y trabajo, además, en la vida cotidiana se piden diferentes favores, se comparten comida, etc.

Desde 1925 Marcel Mauss (1971), en el Ensayos sobre el don, describió que la reciprocidad es una práctica fundamental en una dinámica de permite la cohesión social. El dar, recibir y devolver son fundamentos sociales absolutamente formales, pues en el actuar para mantener el orden de la dinámica comunitaria recae la supervivencia de la comunidad (Orihuela 2020: 4). Justamente, el elemento que inicia la circulación de elementos es el acto de devolver, con lo cual se genera una constante circulación de diversos de elementos considerados regalos. La reciprocidad comunitaria permite establecer una circulación elementos diversos, como trabajo, comida, protección, cuidado, respeto, solidaridad o conocimiento, en el que todos los individuos se encuentran contribuyendo constantemente (Orihuela 2020).

El aprecio que se le da a estos gestos es muy alto. En el siguiente ejemplo se identificará lo antes referido. Días antes de realizar una boda, quince años o un bautizo, la madre del festejado pide a la futura comadre que la espere en su casa con alrededor de 30 invitadas, porque le llevará una «comida de agradecimiento» (tetlamatzuya). Igualmente, la madre acudirá acompañada de 25 o 30 mujeres, que también le apoyaron en la preparación de la comida, que consiste en mole rojo y tortillas. Aunque pueden

18 Cuernavaca, Mor. Septiembre 2019. 
asistir algunos hombres, la reunión tiene el propósito de agasajar a la madrina y a las mujeres invitadas. Para esto, se elige la hora en la que los hombres no se encuentren en casa y poder estar solas. Al anochecer todas regresan a casa después de haber convivido ${ }^{19}$.

Las mujeres forman redes para generar solidaridad comunitaria. Como ya se ha señalado, ellas han sido las que han podido mantenerse mayoritariamente en el bachillerato, pues se les ha apoyado, al igual que a los varones, para este fin a través de redes comunitarias de manera firme. Se les ofrece comida, se les permite realizar sus tareas. Aunque, esto no impide que los jóvenes realicen actividades para apoyar a sus padres en los tiempos correspondientes, ya sea por la mañana o por la tarde, como ir a ver la milpa, en el caso de los muchachos; y hacer labores domésticas, en el caso de las muchachas.

El caso de Doña Luisa confirma lo anterior. Ella menciona que tiene tres hijos, dos mujeres y un hombre. Las mujeres han estudiado hasta nivel profesional, sin embargo, el muchacho abandonó la escuela. Dice "yo las apoyé en los estudios», pues su papá no quiso hacerlo. Más bien, fueron ellas quienes se emplearon para solventar sus gastos. Las jóvenes, al ser apoyadas por su madre y por las redes comunitarias, pudieron asistir al bachillerato $y$ realizar actividades necesarias para obtener recursos. Además, dice doña Luisa que ella les dio el dinero para realizar algunos pagos en la universidad.

Otro de los cambios sustanciales que se presentan es que las mujeres, con derechos de sus terrenos, asisten a las asambleas comunitarias y son escuchadas. Antes, menciona don Emiliano, quien fue comisario ejidal, las mujeres «no iban porque no las dejábamos ${ }^{20}$. Hoy en día las mujeres exponen sus preocupaciones ante el resto del grupo en las asambleas donde participan de forma activa. Incluso, los hombres piden a su esposa o madre que acuda a la reunión en su nombre, cuando ellos no pueden asistir, para que les informen de los asuntos que se atienden comunitariamente (lbid). Las mujeres plantean problemáticas distintas a las que exponen los varones. Incluso, se refieren a cuestiones más sensibles del acontecer social, como

19 Eudocio Bello. Octubre 2019.

20 Emiliano Domingo, quien fue Ayudante y Comisario Ejidal. Cuentepec, Morelos en años anteriores. Mayo de 2019. las enfermedades provocadas por no mantener las costumbres, o la angustia de que las flores no estén creciendo en los terrenos. Al igual que lo hacen los hombres, ellas comparten sus preocupaciones por la falta de lluvia para la siembra, por el daño que se ha hecho a la tierra a causa del mal manejo de los fertilizantes, o por el interés de las empresas mineras por trabajar en su territorio ${ }^{21}$.

\section{PROFESIONISTA DE CUENTEPEC: LETICIA DOMINGO}

La historia de vida de Leticia Domingo, que abordaré a continuación, permite identificar la inserción de cambios y los rasgos culturales que dan continuidad a la dinámica de transición cultural en Cuentepec. La misma dinámica que puede dirigirse hacia un futuro económico y social más estable para las mujeres.

Leticia es parte de la primera generación de profesionistas de la Comunidad de Cuentepec ${ }^{22}$. Ella estudió la licenciatura en Educación Indígena en la Universidad Pedagógica Nacional (UPN) en la Ciudad de México. Ella refiere que para poder estudiar fuera de su comunidad fue determinante el apoyo emocional y económico que recibió de una amiga de la familia que le dio vivienda y alimentación en la Ciudad de México, por lo que Leticia afirma que las redes de apoyo deben y pueden establecerse también con la gente mestiza. También, fue indispensable el apoyo y reconocimiento de su familia para estudiar la universidad alejada de su casa.

Al año de cursar la carrera, Leticia decidió vivir con su novio, un muchacho del pueblo que cursaba la carrera con ella. Pronto se embarazó. Ante la situación, su novio suspendió sus propios estudios para que Leticia continuara en la universidad. También, la mamá de Leticia salió de Cuentepec para vivir en la CDMX con su hija, y, así, poder apoyarla durante los primeros seis meses de vida del bebé. Mientras Leticia regresaba a casa para alimentar y darle los cuidados maternos al pequeño la abuela lo atendía. También, la pareja de Leticia, trabajaba

21 Maribel Villegas. Septiembre 2019.

22 Leticia Domingo, de forma destacada, también participa en La Radio de Morelos con siete cápsulas que se insertan en la programación habitual de la estación radiofónica. El contenido de las cápsulas difunde el idioma náhuatl. 
para solventar los gastos económicos, además, de mantenerse al cuidado del bebé.

Con apoyo de toda la familia, Leticia se tituló y rápidamente encontró trabajo en la CDMX, donde continuó viviendo durante cinco días de la semana, los días de descaso se trasladaba a la comunidad. Leticia siempre consideró que su hogar estaba en Cuentepec, donde su pequeño hijo se quedó al cuidado y protección de su madre y esposo en su ausencia. Así transcurrió el tiempo hasta que su hijo cumplió siete años de edad. Toda su familia respaldó el trabajo que Leticia realiza fuera de la comunidad. Con los recursos que ella genera con su trabajo, solventa gastos importantes.

Mucho del caso de Leticia puede mostrar la transición socioeconómica y cultural concerniente a las mujeres de los pueblos originarios, donde existen una cohesión social que permanece y una actividad económica que cambia. Es preciso resaltar que la experiencia de vida de Leticia deja ver un cambio con respecto del paradigma que ha dominado el contexto indígena, pues éste no refiere a un cambio de roles, donde ella trabaja y su esposo se queda al cuidado de su hijo. Más bien, refiere a las formas de organización social de la comunidad, donde la dinámica de las relaciones de género muestra que las mujeres se apoyan fuertemente entre ellas. Los varones, por su parte, están más apegados a las labores de la milpa y trabajo remunerado en la construcción. Aún con el apoyo comunitario que se les ofrece, tardan más en concluir sus estudios, incluso, tienden a abandonarlos ${ }^{23}$.

Leticia refiere que no vivió violencia familiar. Tampoco la vive ahora con su pareja e hijo. Constantemente Leticia da muestra del respeto y

23 Si bien, en la comunidad se presentan casos en que las jóvenes estudiantes no concluyen la universidad, Leticia relata que otras familias han tomado su ejemplo y ahora apoyan a sus hijas para que terminen la universidad. Es el caso de la hermana de su pareja. Aunque, en un principio los suegros de Leticia no aceptaban la relación de su hijo por apoyarla en continuar con sus estudios mientras su niño crecía al cuidado de él y su suegra, hoy en día apoyan a su hija para que concluya sus estudios. El caso relatado de Leticia Domingo es semejante al de otras jóvenes en la comunidad. Puedo referirme a una joven estudiante de Educación Indígena en la UPN, quién relató ser apoyaba por su pareja para que fuera ella la que terminara la licenciatura mientras él trabajaba en diversas labores. admiración que siente por sus padres. Asimismo, ella conoce y es transmisora de una historia de valores, trabajo duro, conocimiento ancestral y dolor ante la violencia histórica que su comunidad ha vivido en épocas pasadas, todo esto a través de la transmisión oral de su familia y comunidad.

Una desventaja que se observa para las mujeres que salen de la comunidad temporalmente, es que dejan de estar vinculadas con las problemáticas de la comunidad y aseguran que ellas ya no son aceptadas de la misma forma que las mujeres que permanecen en ésta. La comunidad reconoce que al haber estudiado no pueden ver los problemas internos de la misma forma que las personas que se encuentran contantemente en la dinámica comunitaria ${ }^{24}$.

\section{LA MUJER COMO TRANSMISORA DE LA IDENTIDAD COMUNITARIA EN CUENTEPEC}

Ahora bien, otra pregunta necesaria es ¿qué pasa con las mujeres que no desean salir de la dinámica de sus comunidades? ¿Deben entonces continuar con esa exclusión y discriminación hacía lo étnico? Esta pregunta debe responderse con una negación categórica.

Como ya se ha expuesto, las mujeres en la comunidad trabajan arduamente para mantener el soporte monetario de la familia, además proveen de apoyo, tanto a las labores que realizan sus esposos, como a la que realizan sus hijas. No obstante, su papel ancestral y más contundente en la vida comunitaria es la reproducción y transmisión de las formas tradicionales contenidas en la cultura. Ella es quien permite y se adapta a los cambios, y a la vez, es el pilar de la sociedad que da continuidad a los rasgos culturales que permanecen. Ambos factores, permanencia y cambio, son dinámicos.

Dice, Eudocio Bello, Ayudante del Municipio de Cuentepec, "la mujer es la que continúa con las tradiciones». En la mayoría de los testimonios registrados se tiene a la abuela o a la mamá como figura transmisora de los contenidos culturales. Ellas explicaban a los pequeños el porqué de las tradiciones comunitarias o características de su territorio. La mujer es el pilar de la población de Cuentepec. Maribel Villegas revela una frase que da luz para ubicar a la

24 Leticia Domingo, Cuentepec, Mor. Mayo de 2019. 
mujer a partir del pensamiento de la misma sociedad: «nosotros, para conocer a alguien, no le preguntamos ¿cuál es su nombre? Nosotros preguntamos ¿quién o de dónde es su mamá?». Estas palabras afirman que ella establece en la persona la territorialidad, las redes de apoyo íntimas y la transmisión del conocimiento ancestral. Las mujeres construyen cotidianamente la identidad colectiva, aún con la transculturación que se vive actualmente en la comunidad de Cuentepec.

Las mujeres permanecen en movimiento, con su actuar cotidiano reproducen las actividades culturales y establecen la solidez de la vida tradicional. A las mujeres se les observa en las calles de Cuentepec de forma notoria y activa, transitan con paso suave y, a la vez, firme.

\section{RESULTADOS DE LA INVESTIGACIÓN}

Las dinámicas de cambio son perceptibles y reafirmadas por la comunidad. La transición que se trata de contextualizar en Cuentepec abarca un proceso de cambios observados en un periodo de casi tres décadas derivo, entre otros factores sociales, en las implicaciones sociales de una intensa migración pendular de las mujeres. Asimismo, se integraron variaciones en las dinámicas de la vida cotidiana, que determinaron algunos cambios en lo que se definía como el rol de las mujeres.

Se identificó que las dinámicas culturales de las mujeres no son tan estrictas. Las mujeres participan en labores diversas, se mueven en ámbitos que se suponía estaban asignados a los varones. Las dinámicas de transición se configuran para adaptar las costumbres generadas desde épocas anteriores a las nuevas. Ellas trabajan e ingresan en labores más diversas que las aceptadas por los hombres. Las mujeres, entonces, transforman las estructuras tradicionales de organización comunal, permitiendo que otras actividades se incorporen a las que antes ya realizaban, como lo es el estudio.

Las mujeres de Cuentepec han sido y siguen siendo orgullosas de su cultura. Educadas por la familia dentro de normas sociales éticas, crecen teniendo al trabajo como la principal forma de relacionarse y ubicarse en las redes sociales, las cuales son muy sólidas. Las mujeres se mantienen dispuestas al trabajo para apoyar a sus hijos e hijas para que puedan realizar actividades escolares. Además, fomentan en sus hijas la participación en el trabajo tanto doméstico, como económico o escolar.
De forma que las redes de apoyo y su trabajo constante están permitiendo que la transición social se dé con apego a sus valores comunitarios.

La costumbre de formalizar la vida en pareja sigue siendo estable. Tanto que las mujeres estudiantes y/o profesionistas están casadas o viviendo en pareja, aunque en mayor número de casos sí han esperado para embarazarse. Aunque, continúa la costumbre de la unión libre desde temprana edad.

La identidad en Cuentepec está sostenida por su participación comunitaria, que cumple con los cinco puntos señalados por Giménez: cuenta con atributos caracterológicos, un estilo de vida definido por la vida social, una red personal de relaciones íntimas, el conjunto de objetos entrañables, y la biografía personal construida en la vida comunitaria. Todos son elementos culturales que transmite la mujer comunitariamente. Si bien, no se perderá la identidad comunitaria mientras las mujeres sean portadoras y transmisoras de conocimiento, y establezca las redes y alianzas, sí será dinámica.

\section{CONCLUSIONES}

La situación de las mujeres en Cuentepec nos muestra que su dinámica social está aún marcada por las actividades dictadas por el calendario agrícola: sus labores económicas, sus tiempos de descanso, la unión de las parejas. Se ve como un símbolo de pobreza y vulnerabilidad el que las mujeres trabajen y distribuyan su tiempo y ritmos de vida en torno a la agricultura tradicional y a actividades domésticas. Además, las mujeres, a través de múltiples prácticas cotidianas, fortalecen la cohesión social, hacen posible el mantenimiento, continuidad y reproducción de sus contenidos y significados culturales identitarios. Asimismo, ellas generan acciones para impulsar la transición socioeconómica en Cuentepec.

La exigencia a las mujeres de insertarse en la vida laboral y económica más estable no las obliga a cortar los lazos identitarios con su comunidad de origen, tampoco se plantea que las mujeres deben mantenerse aisladas de los procesos económicos globales. Así, es preciso reconocer los fundamentos en los que las sociedades étnicas descansan su identidad individual y colectiva para aproximarnos a la comprensión de los principios con que se afronta la transición cultural. 


\section{BIBLIOGRAFÍA CITADA}

Alavés Vargas, Mayrén. 2010. El paisaje histórico como referencia para la restauración ecológica de Cuentepec, una comunidad nahua de Morelos. Tesis de maestría en Ciencias Biológicas. UNAM.

Argón Urquiza, María del Pilar y Livia Roxana González. 2011. "La vestimenta en Cuentepec, Morelos». Luis Miguel Morayta Mendoza (coord.), Los pueblos nahuas de Morelos. Atlas etnográfico: 288-292. México: INAH y Gobierno del Estado de Morelos.

Bartolomé, Miguel Alberto. 1997. Gente de costumbre y gente de razón: las identidades étnicas en México. México: Siglo XXI.

Benavides Venegas, Farid Samir. 2011. Justicia en épocas de transición conceptos, modelos, debates, experiencias. España: Institut Català Internacional per la Pau.

Bonfil Batalla, Guillermo. 1995. «Concepto de indio en América: una categoría de la situación colonial». Obras escogidas de Guillermo Bonfil, Vol. 1: 105-124. México, INI-INAH.

Caso, Alfonso. 1948. "Definición del indio y de lo indio». América indígena 8(4): 239-247.

Concejo Nacional de Evaluación de la Política de Desarrollo Social. 2012. Informe de pobreza y evaluación en el estado de Morelos 2012. México: CONEVAL.

Domingo Olivares, Leticia. 2016. Prácticas discursivas en la lengua náhuatl de Cuentepec, Morelos y su influencia en la educación. Tesis de licenciatura en Educación indígena. Universidad Pedagógica Nacional.

Figueroa Figueroa, Cecilia y Rocío Carmen Martina Cortes Popoca. 1989. Análisis psicosocial de la cultura popular del estado de Morelos (el caso de San Sebastián Cuentepec). Tesis de licenciatura en Psicología. Universidad Autónoma del Estado de Morelos.

Gran Diccionario Náhuatl. 2012. Universidad Nacional Autónoma de México. Disponible en: <http://www.gdn. unam.mx>. Fecha de acceso: 29 de nov de 2019.

Giménez, Gilberto. 2010. Cultura, identidad y proceso de individualización. México: IIS, UNAM. Disponible en: <http:// conceptos.sociales.unam.mx/conceptos_final/625trabajo. pdf>. Fecha de acceso: 29 de nov. 2019.

Giménez, Gilberto. 2017. «Interculturación y transculturación desde la perspectiva de la historia». En Gilberto Giménez (coord.), El retorno de las culturas populares en las ciencias sociales: 191-220. México: UNAM.

González Ángeles, Livia R. 2005. Milakuaj: etnografía de la ofrenda a los aires en Cuentepec, Morelos. Tesis en Antropología. Universidad Autónoma del Estado de Morelos.

González Ángeles, Livia R. 2016. «El comadrazgo y circulación de ayuda entre grupos domésticos en Cuentepec, Morelos (México)». Devenir. Debates contemporáneos sobre la familia, 30, enero-junio: 39-54.

Hernández Reyna, Miriam. 2013. "La conquista y el argumento del origen en el interculturalismo». En Guy Rozat (coord.),
Repensar la conquista. Reflexión epistemológica sobre un momento fundador. Tomo I: 91-104. Universidad Veracruzana.

Instituto Nacional de Estadística y Geografía. INEGI. 2020. Archivo histórico de localidades geoestadísticas. Histórico de movimientos de Cuentepec, Temixco, Morelos. Disponible en: <https://www.inegi.org.mx/app/geo2/ahl/>. Fecha de acceso: 2 de septiembre de 2019 .

Landázuri Benítez, Gisela. 2001. Visiones, discursos y percepciones de los actores rurales locales y de los profesionistas. Encuentro y desencuentros en Cuentepec, Morelos. Tesis de licenciatura. Universidad Autónoma de México.

Maestro Martínez, Nubia Montserrat. 2011. La actividad física asignada de por género y su relación con la condición nutricia de los adultos nahuas de Cuentepec, Morelos. Tesis en Antropología Física. Escuela Nacional de Antropología e Historia.

Manrique Rivas, Adriana Carina. 1997. Relación con el desarrollo agropecuario de la comunidad de Cuentepec, Temixco, Morelos con la nutrición de la población infantil. Tesis en Ingeniería de Desarrollo Rural. Universidad Autónoma del Estado de Morelos.

Mauss, Marcel. 1961 [1925]. «Ensayo sobre los dones. Razón y forma del cambio en las sociedades primitivas». Sociología y Antropología. Madrid: Editorial Tecnos.

Mota Díaz, Laura y Eduardo Andrés Sandoval Forero. 2011. "Acción social solidaria, confianza y diversidad cultural en América y Latina». Perspectivas críticas sobre la cohesión social. Desigualdad y tentativas fallidas de la integración social en América Latina: 23-51. Buenos Aires: CLACSO.

Navarrete Linares, Federico. 2004. Las relaciones inter-étnicas en México. Colección La Pluralidad Cultural en México, Núm. 3, José del Val. México: UNAM.

Navarrete Linares, Federico. 2008. Pueblos indígenas de México. México: CDI.

Navarrete Linares, Federico. 2010. «¿Qué significa ser indio en el siglo XIX?». En Miguel León Portilla (coord.), Los indígenas en la Independencia y en la Revolución mexicana: 171-190. México: UNAM-INAH-Fideicomiso Teixidor.

Orjuela Escobar, Luis Javier. 2003. "La influencia de la 'doble transición': aproximación crítica a los dos enfoques de política comparada». Colombia Internacional 58, juliodiciembre: 36-64.

Orihuela Gallardo, María del Carmen. (2020). «Reciprocidad: una práctica comunitaria para superar la COVID-19». Notas de Coyuntura del CRIM 37, junio: 1-7.

Paz Salinas, María Fernanda. 2009. «Viviendo en la escasez. E territorio como objeto de transición para la sobrevivencia». Economía, Sociedad y Territorio, enero-abril, año/vol. IX(029): 33-57. El Colegio Mexiquense, Estado de México.

Real Academia Española: Diccionario de la lengua española, 23. a ed., Disponible en: <https://dle.rae.es>. Fecha de acceso: 4 de septiembre de 2020 .

Senado de la Republica. 2010. «Prohíben el matrimonio infantil». Boletines, 21 de marzo. Disponible en: <http:// 
comunicacion.senado.gob.mx/index.php/informacion/ boletines/44207-prohiben-el-matrimonio-infantil.html>. Fecha de acceso: 12 de septiembre de 2020.

Smeke Zonana, Yemy. 2000. «La resistencia: forma de vida de las comunidades indígenas». El cotidiano, vol. 16, núm. 99, enero-febrero: 92-102. México: UAM. Disponible en: <https://www.redalyc.org/pdf/325/32509909.pdf>. Fecha de acceso: 29 de nov. 2019.

Torres Hernández, José Luis. 2018. Historia local, tradición oral y enseñanza de la historia en la escuela primaria de Cuentepec, Temixco, Morelos. Tesis de licenciatura en Educación indígena. Universidad Pedagógica Nacional. 